\title{
Study on construction control system of continuous rigid frame bridge with corrugated steel webs
}

\author{
Z Y Lu' ${ }^{1}, \mathrm{X} \mathrm{K} \mathrm{Cao}{ }^{2}$, J D $\mathrm{Zhang}^{3}$ \\ ${ }^{1}$ University of Chang'an, Department of highway Nanerhuan Road, Xi'an, China, 710064; \\ ${ }^{2}$ University of Chang'an, Department of highway Nanerhuan Road, Xi'an, China, 710064; \\ ${ }^{3}$ University of Chang'an, Department of highway Nanerhuan Road, Xi'an, China, 710064;
}

\begin{abstract}
In order to improve the management and control level of bridge construction and understand the real-time dynamics of bridge construction in real time, a set of cloud 3D visualization information management and control system is created based on the Internet platform. In view of the characteristics of high precision and great difficulty in the construction control of corrugated steel webs bridge, a set of construction control and control software based on B/S (browser/server) architecture is developed to help managers know the site structure state more intuitionistic and improve the efficiency of construction control and control. The system deeply integrates the two-dimensional plane information with the three-dimensional entity, and realizes the three-dimensional display of information flow by highly restoring the real bridge construction state in the three-dimensional scene. The results show that the system can assist the management personnel to carry out construction management and improve the engineering efficiency through linear prediction. The system can realize the dynamic demonstration of the long span CSW-PC continuous rigid frame bridge 3D model and the graphical display of the construction measurement and control data and progress information.
\end{abstract}

\section{Introduction}

With the implementation of "China Bridge 2025" science and technology plan with the theme of intelligent manufacturing key special project "Intelligent Bridge"1, coupled with the high support of relevant national policies, bridge construction control technology ushered in new development opportunities.

Domestic and foreign scholars have carried out related research on construction control system: By studying bridge construction project management, Son $^{2}$ proposed the importance of collaborative management in project implementation and summarized an internal collaboration evolution model for simulation. On this basis, they proposed that the success of a project requires collaborative practice among different individuals. Anumba $\mathrm{CJ}^{3} \mathrm{et}$ al extended the scope of project collaborative work from design to construction stage, established effective methods to manage design and construction information, and developed a Web-based project information management system. Yuan Shuaihua $^{4}$ of Tongji University, based on the Internet, uses high-speed network to realize real-time monitoring of the construction site, and uses expert decision-making function to assist project control. $\mathrm{Li}$ qiao ${ }^{6}$ et al. from Southwest Jiao tong University developed a construction 1PH(+86):15902985876; E-Mail: 2019121059@chd.edu.cn 2PH(+86):19829395090; E-Mail: 1919915630@qq.com 3PH(+86):13359199356; E-Mail: 2019121003@chd.edu.cn control system for super-large span Bridges based on the adaptive control method, which includes six modules, such as control manufacturing, condition evaluation, parameter correction, state prediction and control decision.

However, the current bridge construction process still has many problems, such as many participants, complex information, difficulty in collaborative management, general shortage of information construction, and lack of in-depth research on collaborative and integrated management for multi-subjects of bridge construction projects ${ }^{7}$.Therefore, the construction control software needs to gradually transform from the form of single function, static demonstration and client to the form of multi-function, dynamic demonstration and browser, and realize the transformation of software integration by combining the technology of multi-software interaction ${ }^{11}$.

Aiming at the characteristics of high precision and great difficulty in the construction control of corrugated steel webs bridge, this paper develops a set of construction visual control system based on B/S (browser/server) architecture. The system deeply integrates the twodimensional plane information with the three-dimensional entity, and realizes the three-dimensional display of information flow by highly restoring the real bridge construction state in the three-dimensional scene. The research results can help bridge construction managers 
know the site structure state more intuitively and improve the efficiency of bridge construction control.

\section{System development technology}

The main functions of the corrugated steel web 3D control visualization information system are as follows:

(1) Collect and summarize the information in the construction process, realize real-time monitoring and correction of data, improve the real-time interaction ability of construction information, so as to realize realtime control of the construction process;

(2) Establish a dynamic display model of the construction process, and display the key information of design and construction in a graphical way to improve the efficiency of information communication in the construction process. The framework of this system is shown in Figure 1.

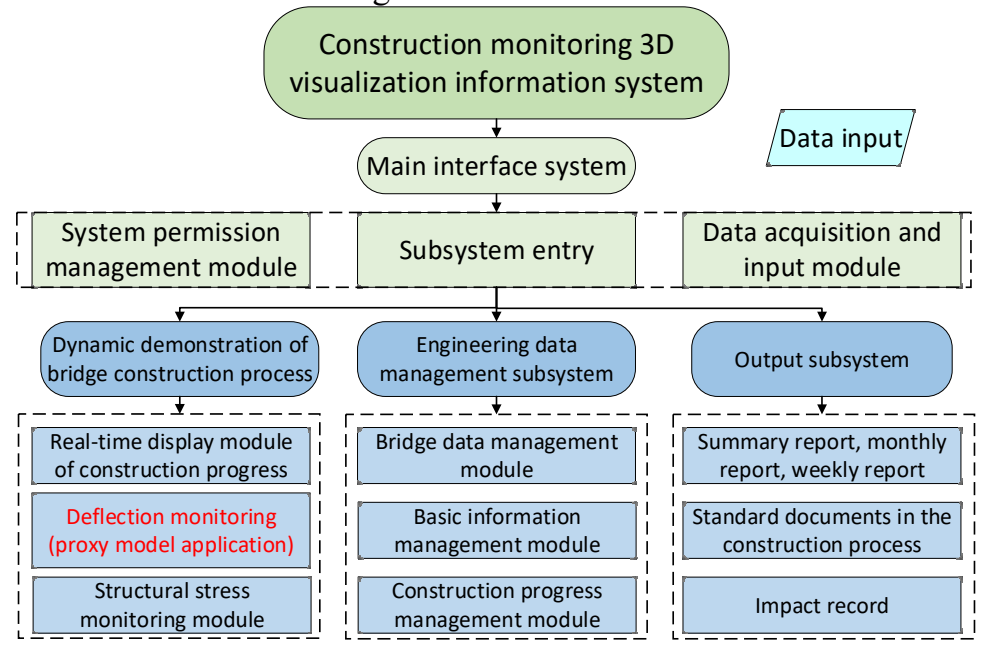

Figure 1. System general frame diagram

This system is developed by Microsoft Corporation.NET development platform, the platform can realize the multi-level function of the software development process, provides a convenient development environment for the Web architecture. Considering that $\mathrm{C} \#$ has the best matching degree with this platform among the mainstream development languages at present and is convenient for later maintenance, $\mathrm{C \#}$ is chosen as the development language.NET development platform and the traditional development platform, the development of a variety of languages, language tolerance is better, in the platform development of the application can be more convenient for multi-system application, and the development of the language to follow the common agreement, improve the development efficiency, so the system.NET system as the development platform, and choose VisualStudio.NET as the system development environment.

At present, software development is mainly divided into $\mathrm{C} / \mathrm{S}$ and $\mathrm{B} / \mathrm{S}$ structure forms (Figure 2). $\mathrm{C} / \mathrm{S}$ structure is called client/server structure. In this structure, tasks are distributed between client and server, and tasks are parsed through machine hardware. The communication between the two ends is very low, but it consumes more network resources, but the task completion level is limited by the machine hardware conditions. With the maturing of the Internet technology, with $\mathrm{B} / \mathrm{S}$ (browser/server) structure design of the system started to gradually get development, the use of this structure can reduce the pressure of the client's hardware, avoid cumbersome client upgrade process, can be directly run through the WEB end, reduces the hardware requirements and operational cost, has the very good applicability, ease and, therefore, The system development chooses to adopt B/S software architecture.

\section{Main girder linear prediction method}

The interface module of MATLAB is developed by C\# language to realize the interaction between the system data and the calculation module of MATLAB, and achieve the purpose of predicting the linear shape of the bridge girder.

\subsection{Interface development}

System design mode: Browser/Sever architecture (Browser/ server architecture).Upload the data using the browser, return to the server to calculate the display results.

Presentation layer development technology: neural network calculation based on Severde, intermediate business module based on $\mathrm{C}$ language, Matlab interface module based on NET Web page.

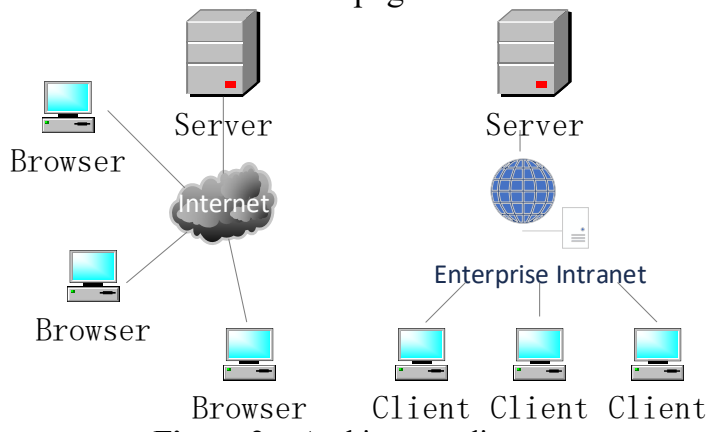

Figure 2. Architecture diagram 


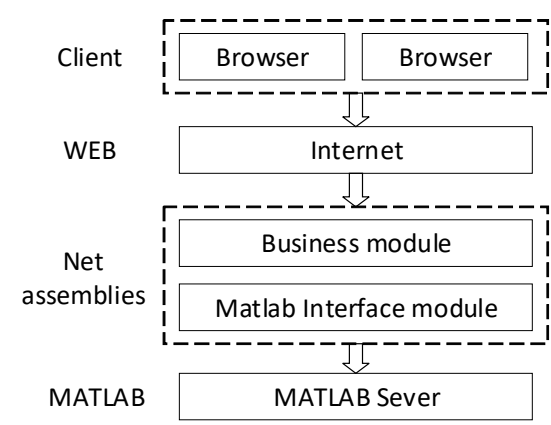

Figure 3. System architecture diagram

MEC-BP algorithm implementation logic: the use of MATLAB grammar in the lowest MEX file.

Computing Engine Component: Under the existing environment and logic, the Matlab ann com class of interface module is developed (variables include network result parameters, workspace path and training data) to realize the communication control of the program. The system architecture is shown in Figure 3.

Neural network constructor: Set Adapt Matrix function is used for setting training data; Set the simulated data using the Set Simulate Matrix function; The reasoning of neural network uses Ann Simulate function.

\subsection{The linear prediction}

In the linear prediction window, by reading the input information in the system, the system data is brought into the finite element model for calculation, and the response output of the predicted linear shape is obtained and displayed through the graphical interface. Input the input parameters, call Matlab interface to realize the predicted value calculation, and display the change rules between the predicted value and the measured value through graphics. It can be seen from the curve change that the fitting accuracy of the linear predicted difference and the measured difference is good, which can meet the error change law, and the predicted results can guide the construction.

\section{The engineering application}

\subsection{Engineering background}

The length of the main bridge is $430 \mathrm{~m}$ $(75 \mathrm{~m}+2 \times 140 \mathrm{~m}+75 \mathrm{~m})$, the roof is $12.65 \mathrm{~m}$, the floor is $6.65 \mathrm{~m}$ wide, the mid-span beam is $3.5 \mathrm{~m}$ high, the pier top beam is $8.5 \mathrm{~m}$ high, and the pier top beam is 1.8 times parabola. The effect drawing of the completed bridge is shown in Figure 4.

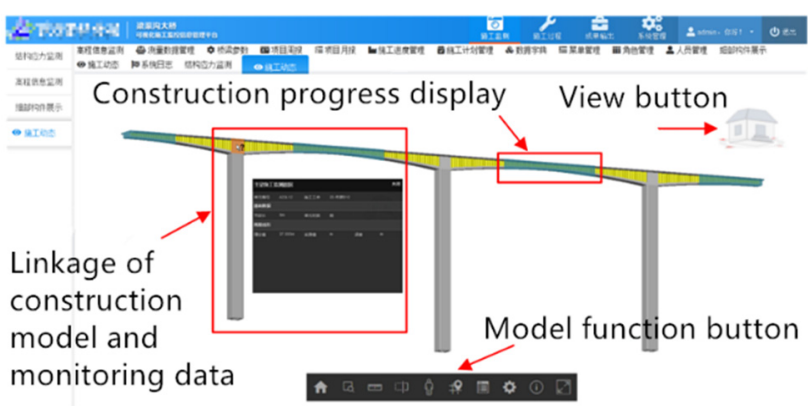

Figure 4. Visualized bridge construction model

\subsection{Main system interface}

After the user logs in the main interface, different subsystems can be accessed through the navigation bar on the upper side. In the main system interface, users can view the real-time dynamic display model of the bridge, through which they can understand the basic information of the project, construction progress status, structural component information, etc., and through this interface, they can realize the management of different subsystems.

The main interface system is mainly divided into four areas:

(1) the menu bar, which is mainly used to provide navigation entrance of each subsystem;

(2) Submenu bar: provide the navigation entrance of each functional module of the subsystem;

(3) $\mathrm{TAB}$ display bar, used to easily open the existing interface;

(4) Information window, used to show the current module function information.

The system management interface can set the basic information of the system, the main functions are: the definition of bridge parameters, user rights management, system menu and subsystem name management and system log management. The basic information of the current project can be defined in the bridge parameter definition module, and the construction information can be managed by defining the basic information of the bridge. In the personnel management module to achieve the management of different login accounts, and through the role management module to give the corresponding authority to different accounts.

\subsection{Construction process dynamic demonstration and monitoring subsystem}

\subsubsection{Dynamic demonstration module}

This module uses WebGL technology to realize the 3D dynamic display of the bridge construction model. This technology better solves the shortcomings of the interactive 3D model on the Web platform, such as relying on external plug-ins, unable to be displayed across platforms and poor compatibility. Relying on this technology, 3D models can be dynamically displayed and interacted with data in the browser platform, without relying on additional plug-ins, and can be applied to a variety of platforms. The $3 \mathrm{D}$ rendering and parsing of the 
model is only carried out by the machine graphics card, which is convenient for users to view at any time and improves the convenience of the system.

The main function of the construction model dynamic display module is to conduct dynamic demonstration of the construction process model and view the main construction information of blocks in the construction process, as shown in Fig. 7.Display the 3D model in different directions through the view button, and display the details of the detail components; View the construction status and construction information under different working conditions through the selection bar of working conditions. When the mouse stays over the block, you can view the main information of the block in the current construction stage (construction progress, elevation monitoring value, stress monitoring value, personnel information, etc.). At the same time, the module reserved the external import interface of the model, which can import the 3D model scanned by the high-precision laser scanner and the model established by the third-party modeling software.

\subsubsection{Linear and stress control module}

In order to ensure construction quality and safety of the structure of the bridge construction process, must always grasp the construction of dynamic information, as the important control targets of construction control, linear control and stress management is more important, so the core modules of this system is linear and stress control module, the module is mainly through the graphical interface, according to facilitate managers identify trends, Timely issue control instructions. The functions of the two modules are as follows:
Linear module to manage construction at different stages in the process of theory of different segment elevation, the measured elevation, camber information beforehand, hanging basket, the cumulative displacement deformation, etc., using the unified data template to import of construction control of the measurement results, convenience of data management, as shown in figure 5, the data and graphical display, to show the change trend of theoretical value and the measured values.

In order to ensure the safety of the bridge construction and meet the design requirements, the stress and strain monitoring of the key interface of the main girder and pier during the construction process is also an important part of the construction control. Due to the influence of the instability of the reinforced concrete material itself, the inaccuracy of the parameters selected by the model, the complexity of the construction condition and the inaccuracy of the calculation software on the actual simulation, the actual stress measured in the construction process is difficult to be exactly the same as the stress calculated by the theory.

Therefore, error determination, analysis and processing of the monitoring results should be carried out during stress testing, and timely adjustment should be made to control the difference between the theoretical stress and the actual stress within the specified range, so as to ensure construction safety and structural reliability. This module can manage the stress information of measuring points in each construction stage, including the theoretical value, measured value and early warning value of each stress measuring point, and dynamically display the change rule of data in a graphical way, and provide decision-making basis for managers through data comparison.

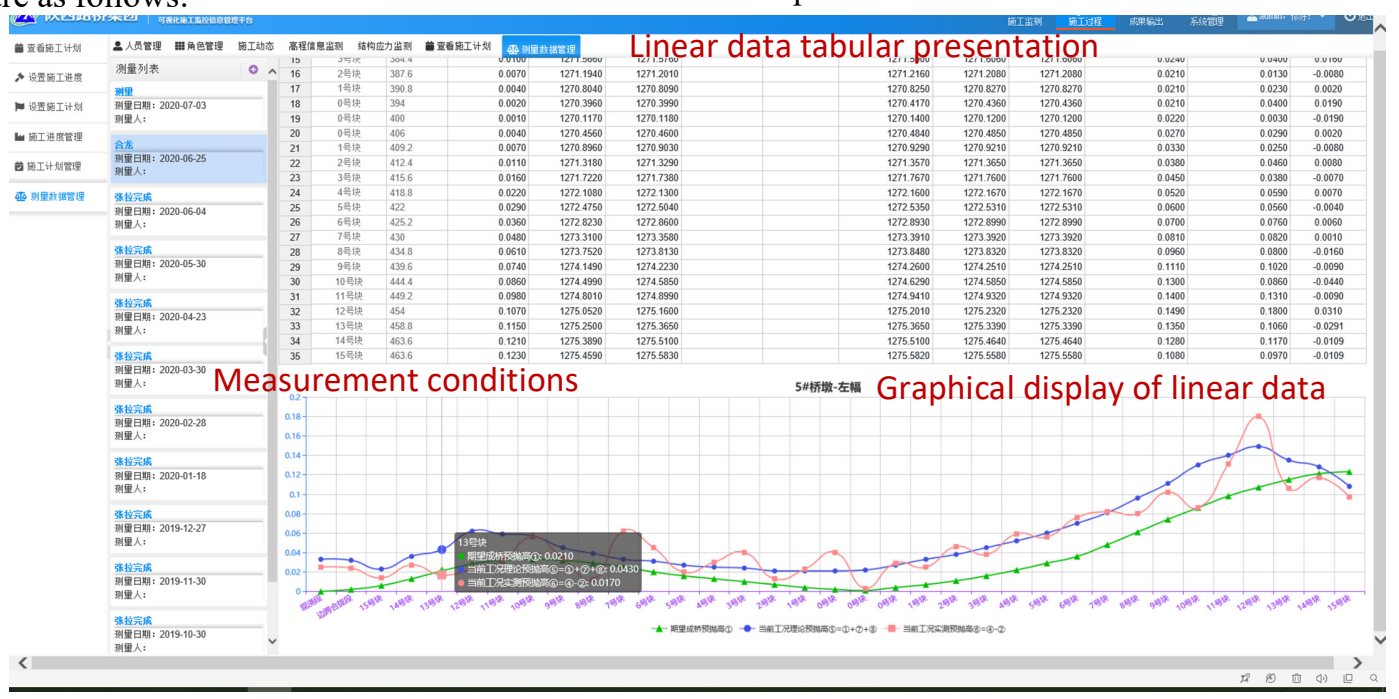

Figure 5. Linear control interface

\subsubsection{Engineering data management subsystem}

Because the system manages a project as a separate project, various information generated in the project needs to be uniformly managed and summarized under different projects. This subsystem mainly manages the following two aspects of information:
(1) Construction schedule and plan: the system can manage the construction schedule and plan information, by clicking the left navigation bar, enter different management projects, in the construction schedule project can fill in the corresponding start and finish dates of different processes, and finally form a horizontal chart.

(2) Stress and elevation data: The system manages the elevation and stress data in construction, and can input the design value, measurement value and other information of 
corresponding elevation measuring points in Excel through batch copying, which improves the input efficiency.

\section{Conclusion}

In this paper, through the independent development of CSW - PC continuous rigid frame bridge construction control $3 \mathrm{~d}$ visualization of information system, the system development technology and each module function have carried on the explanation and demonstration, this paper introduces the construction model of three-dimensional dynamic display dynamic monitoring results and construction data, and applies the conduit bridge beam linear prediction and visualization of construction control, good results have been achieved.

(1) The system realizes a high degree of information flow collection in the construction process, improves the control efficiency of all parties involved in the construction, and achieves good engineering application results.

(2) At present, many functions of the 3D visualization system of construction control and control have not been improved, and the subsequent automatic collection of construction information, real-time import of 3D model, dynamic demonstration of construction model and other aspects need to be improved.

\section{References}

1. Z X Gang, L Gao, M Junhai, W H Bo, F B Yong and G Yuan 2016 J. Present situation and prospect of bridge technology in China. Chinese science bulletin. 61(Z1) 415-25

2. S Jeongwook and M R Eddy 2011 J. Evolution of collaboration in temporary project teams: An AgentBased modeling and simulation approach. J. Constr. Eng. M. 8137

3. Anumba C J, Pan J and Issa R R A $2008 \mathrm{~J}$. Collaborative project information management in a semantic web environment. Engineering Construction and Architectural Management. 15(1) 78-94

4. Y S Hua, S Bin and R Xiao 2008 J. Journal of China \& Foreign Highway. (01) 73-76

5. Y S Hua and R Xiao 2007 J. Research on Intelligent Bridge Construction Control System Based on Network. Journal of Tongji University (Natural Science Edition). (06) 734-738

6. L Qiao, B Y Zhi and Z Q Hua 2009 J. Research on whole-process adaptive construction control system based on geometric control. Chinese Journal of Civil Engineering. 42(07) 69-77

7. Q Q Long 2019 Research on construction control of prestressed concrete continuous girder bridge based on BIM pp 147

8. M Min 2018 Development and application of construction control system for long span continuous girder bridge pp 56
9. Z B Tao 2015 Study on intelligent construction control technology of continuous rigid frame bridge pp 91

10. C De Wei, W X Tong and C W Yun 1998 J. Cablestayed bridge construction control visualization software. Journal of Tongji University (Natural Science Edition). (05) 537-541

11. Z Ju and $\mathrm{H} \mathrm{Q} \mathrm{Wu} 2018 \mathrm{~J}$. Research on Visual Management Method of Bridge Construction Schedule Based on Aerial Panorama. Highway. 63(11) 127-131 